\title{
A validation procedure for a LADAR system radiometric simulation model $^{1}$
}

\author{
Brad Leishman, Scott Budge and Robert Pack \\ Center for Advanced Imaging Ladar \\ Utah State University, Logan, UT 84322-4170
}

\begin{abstract}
The USU LadarSIM software package is a ladar system engineering tool that has recently been enhanced to include the modeling of the radiometry of Ladar beam footprints. This paper will discuss our validation of the radiometric model and present a practical approach to future validation work.

In order to validate complicated and interrelated factors affecting radiometry, a systematic approach had to be developed. Data for known parameters were first gathered then unknown parameters of the system were determined from simulation test scenarios. This was done in a way to isolate as many unknown variables as possible, then build on the previously obtained results. First, the appropriate voltage threshold levels of the discrimination electronics were set by analyzing the number of false alarms seen in actual data sets. With this threshold set, the system noise was then adjusted to achieve the appropriate number of dropouts. Once a suitable noise level was found, the range errors of the simulated and actual data sets were compared and studied.
\end{abstract}

Predicted errors in range measurements were analyzed using two methods: first by examining the range error of a surface with known reflectivity and second by examining the range errors for specific detectors with known responsivities. This provided insight into the discrimination method and receiver electronics used in the actual system.

KEYWORDS: laser sensors and systems, validation, LADAR, simulation, radiometry

\section{INTRODUCTION}

LadarSIM 3.0 is a Matlab and Simulink-based LADAR system simulator designed and developed by the Center for Advanced Imaging LADAR (CAIL) at Utah State University. It is a tool for system analysis, error source modeling, and specifically assists in the design and development of new LADAR systems using pulsed lasers and energy-detection receivers. Recently, as part of the latest software version release, the radiometric simulation model underwent substantial improvements and feature additions [1]. Therefore, a significant validation effort was required in order to verify the accuracy and fidelity of the additions to the software package.

In the validation process actual ladar sensor data needs to be compared to the results of the simulation. In order to do this, controlled experiments which would be easily recreated in the simulation environment needed to be performed to collect data from the hardware LADAR system. The objective of the experiments was to gather data from flat surface returns of varying reflectivity at several different ranges. Three varying sized cubes were used so that these tests could be easily recreated in the simulation and radiometric issues analyzed. Because all of the analysis is done on and around the target surfaces, little attention was dedicated in re-creating the remainder of the scene. After the surfaces were placed in the appropriate positions and distances in the simulated scenes, they were also assigned a reflectivity value and reflectivity model that best described the interaction of the laser pulses with them.

To model the actual ladar system in the simulation, a systematic approach needed to be developed in order to adequately verify the performance and results. This systematic approach involves collecting all known system parameters and

${ }^{1}$ Approved for public release; distribution is unlimited. 
characteristics, using the simulation to estimate unknown or uncharacterized parameters, and examining range error results for surfaces with known reflectivity and for detectors with known responsivity. These range errors can then be compared and analyzed against the actual sensor system results. At the completion of this process, necessary information is gathered from the results and system parameters may be modified and the process repeated.

\section{PARAMETER COLLECTION}

The first step in recreating the test experiments in LadarSIM was to collect all the necessary parameters to model the ladar system. The actual sensor used in these validation efforts consisted of a unidirectional scanning focal plane array in which a derivative crossover discrimination method was used to determine range-to-target measurements [2].

Because this system is competition sensitive, actual system descriptions or specific capabilities will not be discussed in this paper. However, the general outline along with key validation issues and challenges will be presented.

Usually most of the necessary simulation modeling parameters can be found in the respective design and development documentation. For the cases where specific system documentation is not available, LadarSIM offers several bypass and ideal buttons to allow the user to not include or to define ideal settings.

Often specific electrical bandwidths of components in a system are not known but a noise equivalent bandwidth (NEB) is. In this case, the electrical bandwidths of the detector, transimpedance amplifier (TIA) and power amplifiers may be adjusted in order to obtain the desired NEB. For systems which make use of a detector array, responsivity measurements are normally made for each individual detector in the array. Ideally, all of the detectors in the array will react equivalently when illuminated by light, but this is never truly the case. Because of this, LadarSIM allows the user to define and input a custom detector responsivity file which contains the detector specific responsivity information for each detector. Making use of this feature allows the simulation to produce a more realistic radiometric data set. Another customization feature available in LadarSIM is the ability to import a laser pulse file. By default the emitted energy output by a laser pulse is modeled by an ideal Gaussian shape. However, if the actual laser energy has been measured and is available to the user, the simulation can make use of a digitized pulse shape in the construction of the simulation waveforms. This leads to more realistic signal waveforms and better predictions of the radiometric errors.

\section{USING THE SIMULATION TO ESTIMATE UNKNOWN PARAMETERS}

After all known hardware parameters have been collected and input into the simulation, there may be still some parameters which must be estimated by the user. For these cases, the simulation can become a useful tool in helping the user estimate and predict certain unknown parameters when used properly. Typical parameters which require user estimation are the discrimination method threshold setting, system noise level and atmospheric and material characteristics. In these cases, the false alarm and dropout ratios of the actual sensor data can offer clues to the proper remaining simulation settings. A method explaining how to use the simulation as an estimation tool is presented in the following sections.

\subsection{Probability of False Alarms}

Depending on the discrimination method used, there will either be a detection or arming threshold level to be determined. The setting of this threshold will directly affect the number of false alarms seen in the data. The simulation false alarm model is dependant upon the base system noise level, the system NEB ( $\left.B_{s y s}\right)$, and the threshold to noise ratio (TNR) according to the relationship [3].

$$
P_{f a}=1-\exp (-\Delta t \cdot \Lambda)
$$

Where $\Delta t$ is the time interval (gate) that the detector is receiving energy measured in seconds and $\Lambda$ is the false alarm rate defined as [4]

$$
\Lambda=\frac{B_{s y s}}{\sqrt{3}} \cdot \exp \left(-\frac{1}{2} \cdot T N R^{2}\right)
$$


From the parameter collection step, it is assumed that the base noise level and bandwidth of the system have been appropriately set. The base system noise level only includes noise contributions of the system itself independent of the environment or signal. This leaves only the threshold that can be manipulated in order to match the desired false alarm ratio.

False alarms are defined as detections caused by anything besides the reflected energy off of the scene objects. In a realistic environment this may be due to hardware malfunction or atmospheric particles. Because the sensor treats every return the same despite what lead to causing it, it becomes increasingly difficult to identify what data are false alarms and what are legitimate object returns as ranges equal or exceed the range to targets. The only data points which can unequivocally be declared as false alarms are the ones which occur at the ranges in between the sensor and the closest scene returns. By adjusting the threshold level, the user can match the number of false alarms which occur in this region. Figure 1 and Figure 2 show an example of this process. These figures show point clouds as viewed from the side, and at a significant distance from the sensor. As seen in the figures, there are countable false alarms which occur before the returns of the scene. This is the only region where false alarms can be identified accurately.

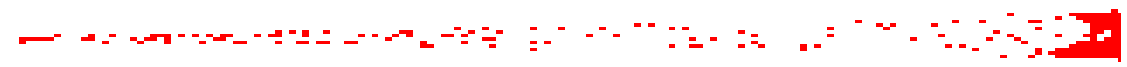

Figure 1. Example point cloud from actual sensor. (125 false alarms before scene)

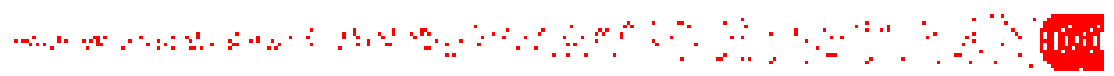

Figure 2. Simulated point cloud with matched false alarm ratios. (124 false alarms before scene)

Because false alarms are range dependant, it is best to match the false alarm ratios at varying distances to the scene with multiple data sets. After performing this analysis at different distances the optimal threshold setting for all ranges can then be determined.

\subsection{Probability of Dropouts}

Once the threshold has been set from the false alarm analysis, the probability of dropouts can be used to determine the total system noise level. The number of dropouts seen in a data set depends on the magnitude of the signal at the detection point $\left(v_{d}\right)$, the voltage threshold $\left(v_{t}\right)$, the total system noise level $\left(\sigma_{d}\right)$ and the laser pulse amplitude jitter ( $\sigma_{A}$ ). This statistical estimation can be described according to the following equation using the standard error function $(\operatorname{erf}())[3]$.

$$
P_{d}=\frac{1}{2} \cdot\left(1+\operatorname{erf}\left(\frac{v_{d}-v_{t}}{\sigma_{d} \cdot \sqrt{2} \cdot \sqrt{1+\sigma_{A}{ }^{2} \cdot\left(\frac{v_{d}}{\sigma_{d}}\right)^{2}}}\right)\right)
$$

In order for a detection to be made, the voltage of the signal at the detection point must exceed the defined threshold level. The signal to noise ratio is often used as the metric in predicting how frequently dropouts will occur. The signal to noise ratio is defined as the ratio between the signal at the detection point versus the total noise present in the system 
at that same point. The higher the signal to noise ratio the less frequently dropouts will occur. For threshold detection systems the peak of the signal is defined as the point of detection because a detection could occur anywhere along the signal up to the peak. The total system noise level includes all sources of the base system noise used in the probability of false alarm calculation along with shot (signal) noise, backscatter and solar background effects. Because the threshold level was set in the previous step the only things left to alter in order to match the probability of dropouts are the signal-dependent noise level and the laser pulse amplitude jitter.

The laser pulse amplitude jitter describes the amount of voltage jitter present on a ladar shot of the defined laser. This jitter greatly affects the form of the probability of dropouts vs. range curve. Figure 3 shows the effect that this parameter has on the probability of dropout simulation estimations. When a target is at close range, the probability of dropouts is largely influenced by the laser amplitude jitter. As the range increases, the distance begins outweighing the jitter in determining the probability of dropout. Therefore, the large and small jitter estimation lines eventually approach one another and cross at some point as shown in Figure 3. Since the actual sensor parameters were used to generate these curves, the actual dropout percentages have been removed in order to not reveal the capabilities of the competition sensitive sensor.

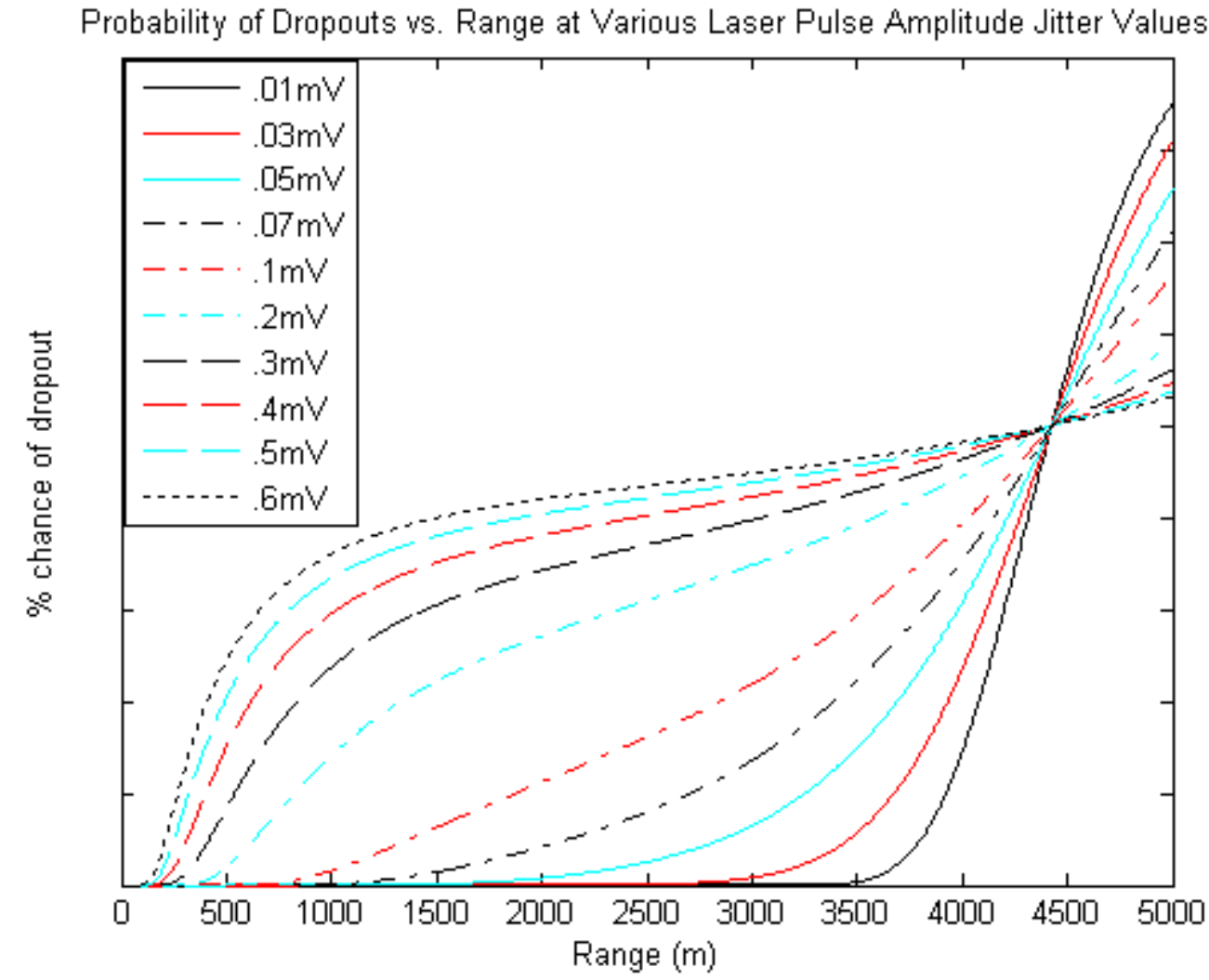

Figure 3. Laser pulse amplitude jitter effects on the probability of dropout estimate curves.

Since laser jitter is usually a difficult parameter to characterize, often the user will need to estimate this. Figure 4 shows a plot of various laser pulse amplitude jitter levels along with the actual probability of dropout percentages (actual numbers have been removed) obtained from the four test scenarios. As seen in the plot, the best curve fit to the actual data is a laser amplitude of about $0.01 \mathrm{mV}$. Because these curves are also affected by the amount of noise in the system an iterative process is usually necessary in order to obtain a solid estimate for both the laser jitter and the system noise level. 


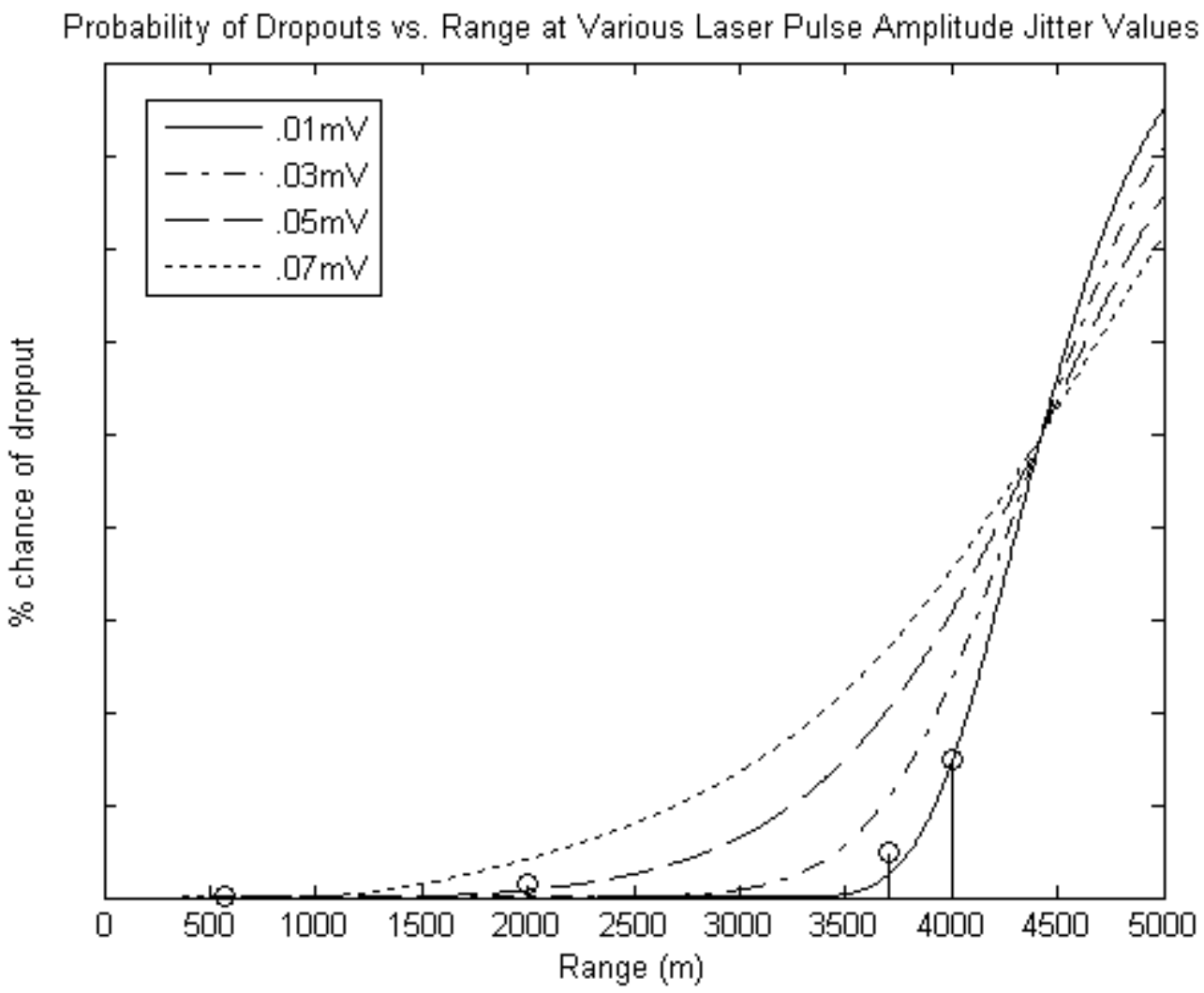

Figure 4. Laser pulse amplitude jitter estimation curves with actual sensor results marked by the stem points.

In order to use the simulation to determine the proper amount of system noise, simulated scenes needed to be generated with range variations and angles of incidence approximately equivalent to the sensor data. Using the same test scenarios the scenes were generated and the necessary noise was input into the system in order to achieve the actual data set probability of dropouts. To increase the total noise, the backscatter and solar background contributions were increased. This was done by adjusting the aerosol, visibility and spectral irradiance values which describe the atmosphere. The motivation for selecting these parameters to be modified was that these values are not typically available through direct measurement so their specific settings are not usually known. The reflectance parameters of the ground were estimated to have a diffuse reflectivity model with an average of 30 percent reflectivity given the surface cover of the test site.

With the appropriate system noise level set and similar scenes created, LadarSIM becomes an effective tool in predicting the dropouts seen in actual data. Figure 5 shows a range image obtained from the sensor data. Figure 6 shows a comparison range image using a simple flat plane as the ground with the adjusted detector noise level.

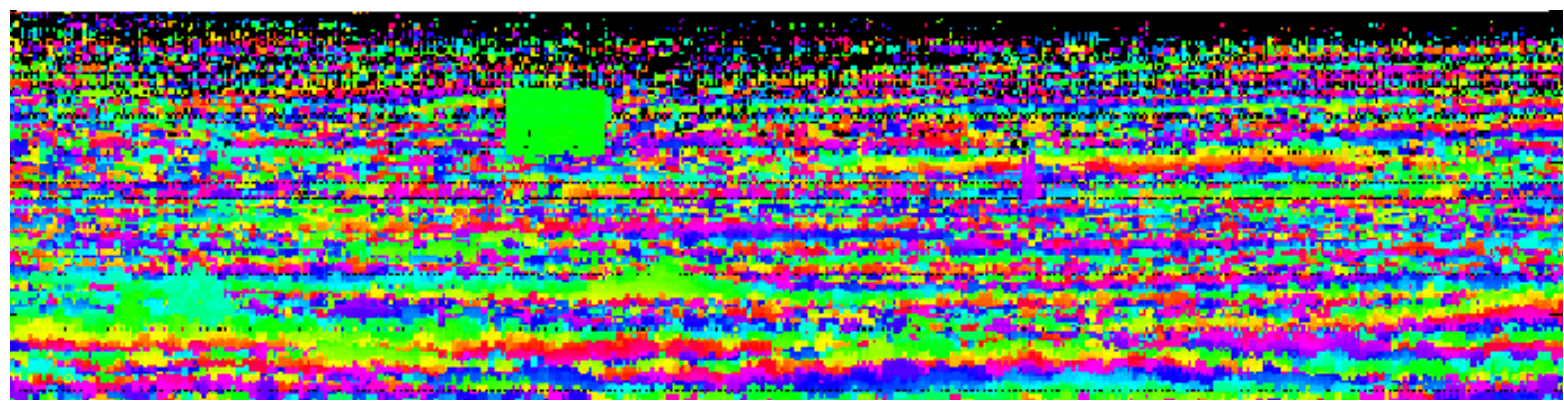

Figure 5. Range image of actual sensor data. (14.91\% of points were dropouts) 


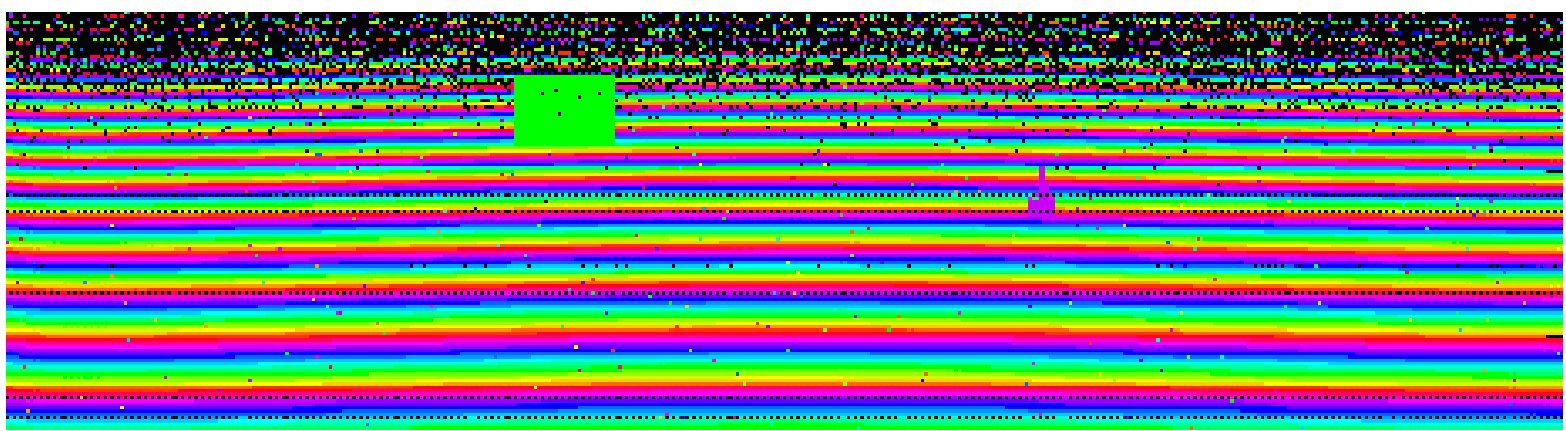

Figure 6. Range image of simulation data using a simple flat plane to model the ground. (15.32\% of points were dropouts)

The horizontal stripes of dropouts observed in Figures $5 \& \mathbf{6}$ are caused by the fact that certain detectors are more susceptible to missing detections. This is because of the material properties of the avalanche photodiodes themselves. Detectors with low responsivity or quantum efficiency are not able to collect as much energy as the same detector area with higher responsivity. This can lead to more dropouts for a certain detector. In order to model this, LadarSIM accepts a custom detector file which contains the responsivity measurements of each individual detector in an array. As seen in Figure 5, there are a few detectors near the middle of the image and one near the bottom with low responsivity. This leads to rows of dropouts where someone would not expect to see them. With the custom detector file loaded, LadarSIM effectively simulates such phenomena, as shown in Figure 6.

\section{RANGE ERROR ANALYSIS}

Range errors are defined as the difference between the actual distances and the system-measured distances. Range error is traditionally described in terms of a variance or standard deviation. This paper will use the latter. Most ladar sensors are vulnerable to many causes of interference and therefore range errors become a measure of the accuracy and repeatability of the system under certain conditions. In the simulation environment, each measurement starts off exact, then a probability of dropout, walk error and range error are applied to simulate the radiometric phenomena seen in actual systems. Walk error is only apparent when a simple detection threshold technique is being used. The amount of this error is dependant upon the intensity and shape of the return signal waveform. The amount of range error $\left(\sigma_{R}\right)$ applied to a respective detection at time $t_{p}$ depends upon the speed of light $(\mathcal{C})$, the predicted noise level $\left(\sigma_{d}\right)$ and the slope of the pulse at the point of detection $\left(\frac{d}{d t} v\right)$ according to the following equation [2].

$$
\sigma_{R}=\frac{c}{2} \cdot \frac{\sigma_{d}\left(t_{p}\right)}{\left|\frac{d}{d t} v\left(t_{p}\right)\right|}
$$

This equation shows that the higher the slope is at the timing point the better the range estimation will be. This is equivalent to saying that higher signal to noise ratios give better range estimates. Moreover, the higher the system noise level is the worse the range estimations will be. This is one of the primary motivations to reduce noise in Ladar systems.

In order to compare the simulation predicted range errors to those of the actual data, the range error of the sensor data must be determined from its range image. This process consists of identifying a region of points of approximately similar range then determining the standard deviation of the range measurements. In order to keep consistency in the data collection, the same group of points were also used to determine the range standard deviation of the simulation data. The range errors of the sensor and simulation data were compared and analyzed in two categories. First, the range errors were compared from points of surfaces of known reflectivity. Then, they were analyzed for detectors with known quantum efficiency. The specifics involved and issues discovered will be presented in the next two sections. 


\subsection{Range Error of a Surface with known Reflectivity}

Three various sized cubes were used in the data collection test scenarios. These were selected because they would provide flat surfaces to determine accurate range errors and would be easily recreated in the simulation environment. Figure 7 shows the setup of these cubes in the simulation environment. The actual cubes had faces painted white or gray. Depending on which side was facing the sensor, the reflectivity values of the cubes inside the simulation were modified in order to model the different paints. The reflectivity of the white paint was estimated to be about 80 percent while the reflectivity of the gray was estimated to be about 60 percent. Both materials were assumed to follow a diffuse reflectance model in the simulation.

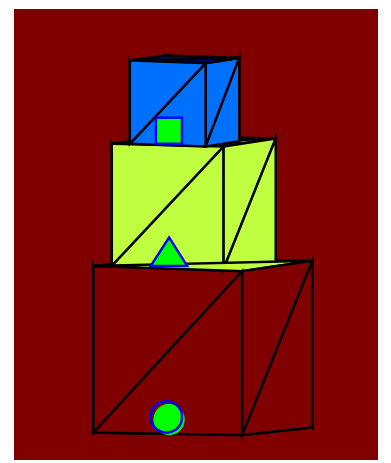

Figure 7. Simulation modeled cubes used for the validation tests.

When the analysis and comparisons of the actual to the simulated data was performed, the simulation range error estimates were consistently less than the actual results. This can be seen in Figure 8 which plots the range error of the actual data and the simulated data vs. range (note: range error values are competition sensitive and have been removed ). Section 4.3 will discuss the possible causes of this range error discrepancy.

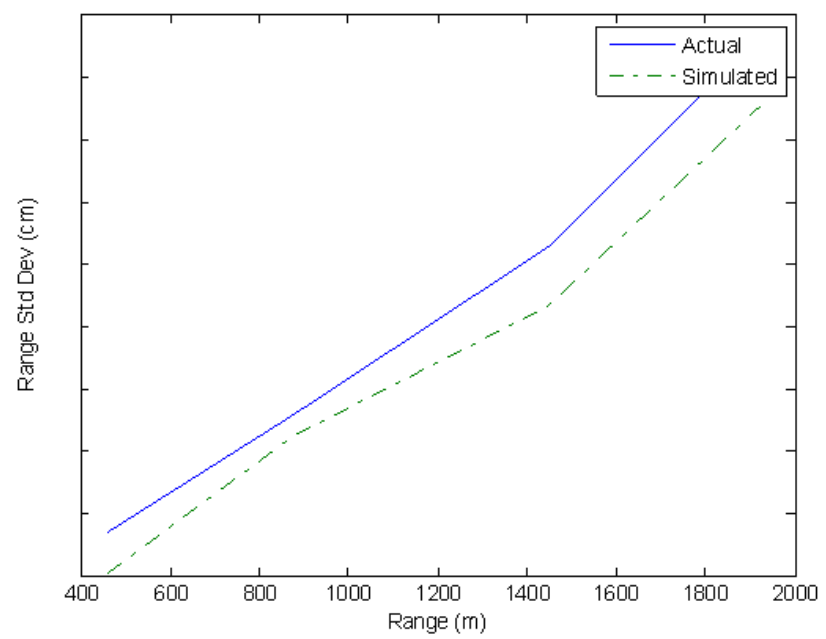

Figure 8. Comparison of range errors between the sensor and the simulation.

\subsection{Range Errors of Detectors with known Responsivities}

The second stage in validating the simulation range errors was to analyze the range error standard deviation for a single detector with known responsivity. Because the discrimination method used in the actual system is theoretically amplitude invariant, it was assumed that range error estimations shouldn't be affected much by various detector responsivity values. A higher detector responsivity could lead to more discernable signal over noise resulting in a better estimate. Although this is true, the variation of the detector responsivities was too small to see a discernable connection. Since detector arrays are created so that all detectors in the array have similar responsivities, this will usually be the 
case for amplitude invariant systems. Figure 9 shows a comparison of the range errors vs. responsivity of the sensor and the simulation data (note: range error values are competition sensitive and have been removed).
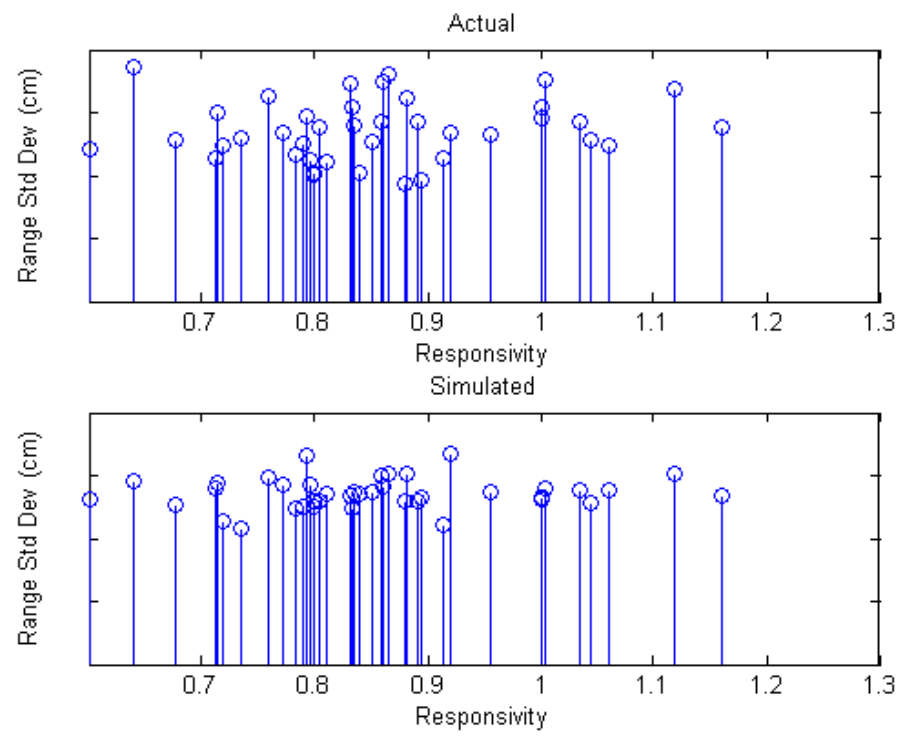

Figure 9. Range error vs. responsivity measurement comparison.

If a similar simulation was preformed for a system susceptible to walk error (amplitude variant), a definite correlation between detector responsivity and range error could be seen. Detectors with greater responsivity resulting in a higher amplitude return signal will bias the range measurements so they seem closer to the sensor than those collected by detectors with lesser responsivity. This phenomenon is more commonly seen by surfaces with varying reflectivity, but similar effects can be seen with varying detector responsivities. Doing error analysis with respect to responsivities is sometimes difficult to interpret because responsivity varies with wavelength, position on detector, angles of incidence, time, temperature of radiometer, source temperature, environmental temperature, incidence, polarization, degree of coherence, and relative humidity [5]. Though similar phenomena were present in the sensor data, we considered the resulting variations negligible.

\subsection{Simulation Range Error Inaccuracy Causes}

As mentioned previously, the simulation range error depends on two things: the slope of the pulse and noise at the point of detection. There are various issues in both of these areas that need to be taken into consideration when discussing the possible causes of inaccuracy of the simulation range error estimates. Many discrimination methods used in declaring detections are individually tailored to specific ladar systems. LadarSIM offers three generalized discrimination methods that are often used in ladar receivers. These generalized methods may not be exactly the same as those designed for a specific system and therefore may result in somewhat erroneous predictions.

The actual shape of the pulse will affect range error by altering the slope of the pulse at the detection point. Because of this, LadarSIM allows the user the ability to import a custom pulse file which may describe a particular laser pulse more accurately than an idealized Gaussian shape. The shape of the return signal waveform is also greatly dependent upon the magnitude of energy which is returned. This depends on properly estimating or measuring the emitted laser power parameters, receiver and transmitter optical efficiencies, atmospheric conditions and reflectance parameters of the objects which the laser beams interact with. Errors or inaccuracies in any of these areas will lead to misleading return signal waveforms causing an over or underestimation of the accompanying range errors.

The range error is also affected by the noise at the detection point. This is a complex calculation which depends on both atmospheric and system-level parameters. Although the noise simulation modeling of LadarSIM encompasses many noise sources, it does not attempt to model every possible known source. Crosstalk, thermal effects, drift and 
systematic errors are not modeled by in the simulation. However, they can be lumped together and included as constant noise sources in the simulation if desired.

Other system specific design issues can also play a role in range error estimation. If a system is susceptible to optical misalignment so not all laser energy falls squarely on the active detector surface area, range errors can be increased. Also, for systems utilizing a detection threshold to declare timing points, the range error must be thought of as a combination of the error due to noise and walk error. All of these issues must be taken into account when analyzing the results of LadarSIM simulations so that they are not misinterpreted.

\section{CONCLUSION}

Through the validation work presented in this paper, LadarSIM has been shown to be an effective tool in predicting radiometric errors such as false alarms, dropouts and range errors. As discussed above, the fidelity of the simulation results achieved is directly correlated with how well the ladar system is modeled by the user. Since random processes are involved, the prediction of the radiometric errors is meant to be an approximation and not exact. This is due to the fact that not all phenomena is perfectly understood or modeled.

When system parameters are not known, they can be estimated with the help of the simulation results following the procedure outlined above. A practical systematic method was presented in order to minimize the parameter interrelation and build on the previously determined values. When the system and simulation are properly understood, LadarSIM is an effective tool in the development and analysis of ladar systems.

\section{REFERENCES}

[1] S. Budge, B. Leishman and R. Pack, "Simulation and modeling of return waveforms from a ladar beam footprint in USU LadarSIM". Proceedings of SPIE, Laser Radar Technology and Applications XI, 2006.

[2] P. P. Tarmo Ruotsalainen and J. Kostamovaara, "A wide dynamic range receiver channel for a pulsed time-of-flight laser radar," IEEE Journal of Solid-State Circuits, vol. 36, No. 8, 2001.

[3] R. W. Byren, Laser Rangefinders, vol. 6. Washington: SPIE Optical Engineering Press, 1993.

[4] C. W. Helstrom, Elements of Signal Detection and Estimation, p. Section 7.3.3. PTR Prentice Hall, Englewood Cliffs, NJ, 1995.

[5] W. L. Wolfe, Introduction to Radiometry. SPIE Optical Engineering Press, 1998. 